\title{
Fuzzy Based Experimental Verification of Significance of Skull Tissue removal in Brain Tumor Image segmentation
}

\author{
D. Jude Hemanth \\ Dept. of ECE \\ Karunya University \\ Coimbatore, India
}

\author{
C. Kezi Selva Vijila \\ Dept. of ECE \\ Karunya University \\ Coimbatore, India
}

\author{
J. Anitha \\ Dept. of ECE \\ Karunya University \\ Coimbatore, India
}

\begin{abstract}
In anatomical aspects, magnetic resonance (MR) imaging offers more accurate information for medical examination than other medical images such as X-ray, ultrasonic and CT images. Tumor segmentation from MRI data is an important but time consuming task performed manually by medical experts. Automating this process is challenging due to the high diversity in appearance of tumor tissue, among different patients and, in many cases, similarity between tumor and normal tissue. One of the reasons behind the inferior segmentation efficiency is the presence of artifacts in the MR images. One such artifact is the extracranial tissues (skull). These extracranial tissues often interfere with the normal tissues during segmentation that accounts for the inferior segmentation efficiency. In this paper, an automated segmentation and lesion detection algorithm for high segmentation efficiency is proposed for abnormal MR brain images. The proposed segmentation algorithm consists of three steps. In the first step, extracranial tissues are removed using morphological operations. In the second step, fuzzy C-means algorithm is used to segment the MR brain images into four groups: white matter, gray matter, cerebrospinal fluid and the abnormal tumor region. Finally, pseudo-colouring operation is performed on the segmented image to detect the abnormal tumor region. The proposed method has been applied to abnormal images from four different types namely metastase, meningima, glioma and astrocytoma.the superior nature of the proposed approach is justified by performing a comparative analysis on skull stripped images and non-skull stripped images. Experimental results suggest that the proposed approach provides an effective and promising method for brain tumor extraction from MR images with high accuracy.
\end{abstract}

\section{Keywords}

MRI, segmentation, fuzzy, extra cranial, segmentation efficiency.

\section{INTRODUCTION}

Radiation oncologists, radiologists, and other medical experts spend a substantial portion of their time in segmenting medical images. Accurately labeling brain tumors and associated edema in MRI (Magnetic Resonance Images) [1] is a particularly timeconsuming task, and considerable variation is observed between labelers. Subsequently, over the last 15 years, a large amount of research has been focused on semi-automatic and fully automatic methods for detecting and/or segmenting brain tumors from MRI scans.

Brain segmentation methods can be broadly categorized as

manual methods and computer-aided semi automated or automated methods [2,3]. In recent years, computer-aided segmentation methods have been developed at a rapid pace to overcome the disadvantages of the manual segmentation methods. These methods are more automatic, objective and the results are highly reproducible [4]. Various computational algorithms, ranging from semi automated (requiring user interactions) to fully automated have been developed [5, 6]. An important application of segmenting the tumor region is in tracking the size of the brain tumor as it responds to treatment [7]. Therefore an automatic and reliable method for segmenting tumor would be a useful tool [8].

The tumors vary greatly in size and position, have a variety of shape and appearance properties, have intensities overlapping with normal brain tissue, and often an expanding tumor can detect and deform nearby structures in the brain giving an abnormal geometry also for healthy tissue. Therefore, in general it is difficult to segment a tumor by simple unsupervised thresholding [9]. Other more successful approaches consider texture information $[10,11]$. Different machine learning (ML) segmentation techniques have been investigated: Neural Networks [11], SVMs (Support Vector Machines) [12, 13], MRFs (Markov Random Fields) [14, 15], and recently CRFs (Conditional Random Fields) [16]. But, as previously mentioned, statistical techniques may not allow differentiation between non-enhancing tumor and normal tissue due to overlapping intensity distributions of healthy tissue with tumor and surrounding edema [17]. Others have introduced knowledge-based techniques to make more intelligent and segmentation decisions, such as in [18].

In this paper, multi-spectral analysis in the form of unsupervised clustering is performed on abnormal MR brain tumor images. Initially, the extracranial tissues are removed from the images. An orderly usage of various morphological operations has removed the extra cranial tissue to a greater extent. In the second step, image segmentation is performed using fuzzy C-means algorithm. A pseudo-colouring operation is performed on these images to detect the lesion region. Different colours have been assigned to different regions namely white matter, gray matter, cerebrospinal fluid and the abnormal tumor region. The segmentation efficiency of the proposed approach is calculated by comparing the results with the ground truth images. FCM based segmentation is also performed on images with extracranial tissues. A comparative analysis in terms of segmentation efficiency clearly reveals the necessity for skull tissue removal in brain image segmentation.

\section{PROPOSED METHODOLOGY}

The proposed methodology of segmenting brain tumor of abnormal brain using the fuzzy C-means algorithm is shown in Figure 1. 


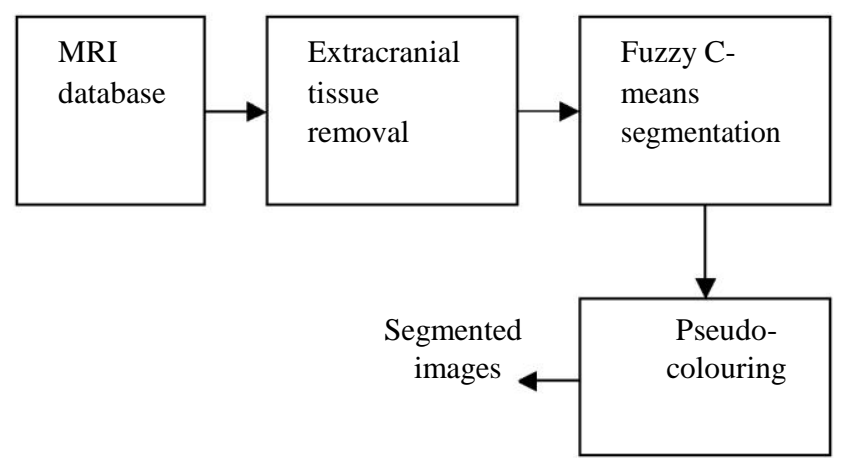

Figure 1. Proposed Methodology

In this work, real time abnormal MR brain images collected from scan centre are used. As a pre-processing step, the extracranial skull tissues surrounding the soft brain tissues are removed using the morphological operations. The connected component analysis combined with erosion operation removes the extracranial tissues to a greater extent. The MR images are then segmented into different clusters using the fuzzy c-means algorithm. An optimum number of clusters are chosen to avoid over-segmentation. In this work, the images are clustered into four groups. Different colours have been assigned to different regions to distinguish their characteristics. The abnormal region is labeled with a different colour to distinguish from other normal brain tissues. The number of colours depends on the number of clusters used. Finally an extensive comparative analysis is performed between the images with extracranial tissues and without extracranial tissues in terms of segmentation efficiency.

\section{MRI DATABASE}

A set of MR brain tumor images comprising of the four tumor types namely meningioma, astrocytoma, glioma and metastase are collected from radiologists. The images used are $256 * 256$ gray level images with intensity value ranges from (0 to 255). Initially, these MRI images are normalized to gray level values from ( 0 to 1) and the features are extracted from the normalized images. Since normalization reduces the dynamic range of the intensity values, feature extraction is made much simpler. Some samples of the MRI database have been displayed in Figure 2.

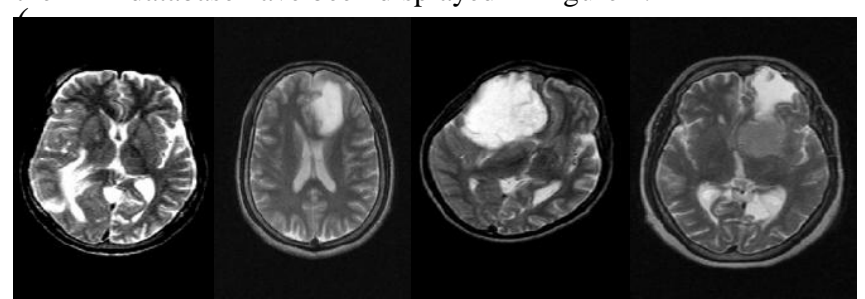

(a)

(b)

(c)

(d)

Figure 2. Sample data set: (a)Metastase (b)Glioma (c)Astrocytoma (d) Meningioma

\section{EXTRACRANIAL TISSUE REMOVAL}

The various steps involved in the removal of extracranial tissues are based on morphological operations.

\subsection{Morphology}

Morphology is a tool for extracting image components that are useful in representation and description of images [19]. The objects in an image are represented by sets in the morphology theory. For binary images each element of the set is a 2-D vector, corresponding to the $(\mathrm{x}, \mathrm{y})$ coordinates of a black (or white depending on the object) pixel. It can be defined as a broad set of binary image operations that process images based on shapes. Morphological operations apply a structuring element to input image, creating an output image of the same size. The morphological operations used are dilation, erosion, opening. closing, etc.

\subsection{Sequence of Extracranial Tissue Removal}

The various steps followed in the extracranial tissue extraction are shown in Figure 3.

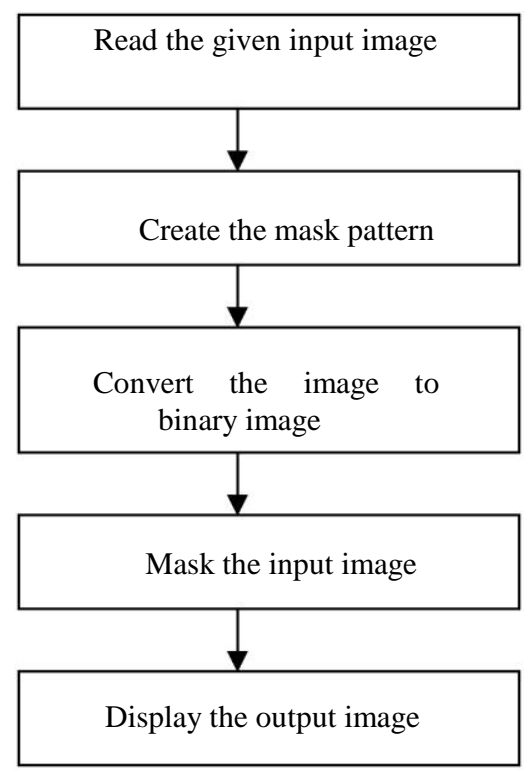

Figure 3. Sequence of extracranial tissue removal

\subsection{Algorithm}

\section{Step 1 : Generation of mask}

The first step after reading the image is the generation of a mask. Two basic morphological operations namely erosion and filling are used in this work to generate the mask.

\section{a) Erosion:}

The main function of this operation is to remove the pixels on object boundaries. The rule given below defines the operation of the erosion operation.

"If every pixel in the input pixel's neighborhood is on, the output pixel is on. Otherwise, the output pixel is off".

Erosion operation uses a specified neighborhood [20]. The neighborhood for an erosion operation can be of arbitrary shape and size. The neighborhood is represented by a structuring element, which is a matrix consisting of only 0's and 1's.the shape of the structuring element used in this work is "ball" and the size of the structuring element is 5-by-5.next, a suitable threshold is selected and the pixels above the threshold value are made equal 
to 255 and the pixels below the threshold value are made equal to 0 .

b) Connected component analysis:

A process that "fills" a region of interest by interpolating the pixel values from the borders of the region. This process can be used to make objects in an image seem to disappear as they are replaced with values that blend in with the background area [21]. This function is useful for removal of extraneous details or artifacts. The resultant image after these two operations yield the mask for the corresponding input image has been generated.

\section{Step 2 : Conversion to binary image}

The generated mask is a gray level image. For further analysis, the mask is converted into a binary image.

\section{Step3 : Masking}

The original input image is masked with the binary mask. The masking is achieved by performing a multiplying operation between the original image and the mask. The resultant image is free from the extracranial tissues, which is evident from the output images.

\section{FUZZY SEGMENTATION}

Fuzzy c-means (FCM) is a method of clustering which allows one pixel to belong to two or more clusters [22]. The FCM algorithm attempts to partition a finite collection of pixels into a collection of "C" fuzzy clusters with respect to some given criterion. Depending on the data and the application, different types of similarity measures may be used to identify classes. Some examples of values that can be used as similarity measures include distance, connectivity, and intensity. In this work, the skullstripped images are segmented into four clusters namely white matter, grey matter, CSF and the abnormal tumor region based on their intensity values.

\subsection{Algorithm}

Fuzzy c-means algorithm is based on minimization of the following objective function:

$$
J\left(U, c_{1}, c_{2}, \ldots, c_{c}\right)=\sum_{i=1}^{c} J_{i}=\sum_{i=1}^{c} \sum_{j=1}^{n} u_{i j}{ }^{m} d_{i j}{ }^{2}
$$

$\mathrm{u}_{\mathrm{ij}}$ is between 0 and 1 ;

$c_{i}$ is the centroid of cluster $i$;

$\mathrm{d}_{\mathrm{ij}}$ is the Euclidian distance between $\mathrm{i}_{\text {th }}$ centroid $\left(\mathrm{c}_{\mathrm{i}}\right)$ and $\mathrm{j}^{\text {th }}$ data point.

$\mathrm{m} \epsilon[1, \infty]$ is a weighting exponent.

\section{Step 1}

Fuzzy partitioning of the known data sample is carried out through an iterative optimization of the objective function shown in eqn (16), with the update of membership $u_{i j}$ and the cluster centers $c_{i}$ by:

$$
u_{i j}=\frac{1}{\sum_{k=1}^{c}\left(\frac{d_{i j}}{d_{k j}}\right)^{2 /(m-1)}} ; \quad c_{i}=\frac{\sum_{j=1}^{n} u_{i j}{ }^{m} x_{j}}{\sum_{j=1}^{n} u_{i j}{ }^{m}}
$$

Step 2:

At $\mathrm{k}_{\mathrm{th}}$ number of iteration:

Calculate the center vectors $c_{i}$ with $u_{i j}$

$c_{i}=\frac{\sum_{j=1}^{n} u_{i j}{ }^{m} x_{j}}{\sum_{j=1}^{n} u_{i j}{ }^{m}}$

Step 3:

Update the membership matrix $\mathrm{U}$ for the $\mathrm{k}_{\mathrm{th}}$ step and $(\mathrm{k}+1)_{\mathrm{th}}$ step.

$$
u_{i j}=\frac{1}{\sum_{k=1}^{c}\left(\frac{d_{i j}}{d_{k j}}\right)^{2 /(m-1)}}
$$

\section{Step 4:}

If $\|\mathrm{U}(\mathrm{k}+1)-\mathrm{U}(\mathrm{k})\|<\varepsilon \quad$ then STOP; otherwise return to step 2 .

The threshold value $\varepsilon$ used in this work is 0.01 . This algorithm segments the image into four different regions with the cluster centers and the membership values of each pixel.

\section{PSEUDO-COLOURING}

Pseudo-colouring does form an integral part of image enhancement. As can be seen, hidden features become easily distinguishable after pseudo-colouring. It involves the assignment of colours to a range of greyscales. It is in ones best interest to manipulate these colours to be of varying shades and tones so as to bring out the appropriate detail in images. it is also a method of region labeling in which different colours depicts the four different regions. In this work, four different colours are assigned to the four clusters in order to have a clear differentiation between the four regions.

\section{IMPLEMENTATION}

The MR slices were acquired on a 0.2 Tesla, Siemens magnetom CONCERTO MR Scanner (Siemens, AG Medical Solutions, Erlangen, Germany) from Devaki MRI and CT scans Madurai, INDIA. The scan image was taken with axial, 2D, 5mm thick slice, with a slice gap of $2 \mathrm{~mm}$, with $246 * 512$ acquisition matrix and with the field of view of $250 \mathrm{~mm}$. The T2 (TR/TE of $4400 / 118 \mathrm{~ms}$ ) and T2-FLAIR (TR/TE of 6160/89ms) weighted images were collected using Turbo Spin Echo (TSE) sequences. In this study, 374 abnormal images from four different classes are used. The data set used for this segmentation problem is as shown in Table 1 .

Table 1. Data set for brain tumor segmentation

\begin{tabular}{|l|r|}
\hline Tumor type & $\begin{array}{c}\text { Number of } \\
\text { images/class }\end{array}$ \\
\hline Meningioma & 102 \\
\hline Astrocytoma & 82 \\
\hline
\end{tabular}




\begin{tabular}{|l|r|} 
Metastase & 96 \\
\hline Glioma & 94 \\
\hline $\begin{array}{l}\text { Total } \\
\text { abnormal } \\
\text { images }\end{array}$ & 374 \\
\hline
\end{tabular}

\section{Parameters used:}

In skull removal, "ball" structuring element is used along with the erosion operation. The threshold value ranges from 40 to 80 .

In fuzzy segmentation, the images are segmented into four clusters. The error threshold value is 0.01 . The exponential component value is 2 . In pseudo-colouring, HSV colour model is used to distinguish the four segmented clusters.

The validation of the results is performed by comparing the experimental results with their corresponding ground truth. The experiments are carried out on an IBM PC Pentium with processor speed $700 \mathrm{MHz}$ and $256 \mathrm{MB}$ RAM. The software used for the implementation is MATLAB (version 7.0) [23], developed by Math works Laboratory.

\section{RESULTS AND DISCUSSIONS}

In this work, 374 images from four different brain tumor types are used. Initially, the images are skull stripped and then segmented using clustering algorithm. To show the superior nature of the proposed approach, the results are compared with the segmented output of images with extra cranial tissues. The segmentation efficiency is calculated with the aid of the ground truth images.

\section{Stage 1 results:}

The process of extraction of skull tissues is depicted in Figure 4.

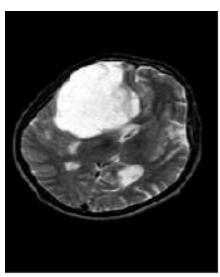

(a)

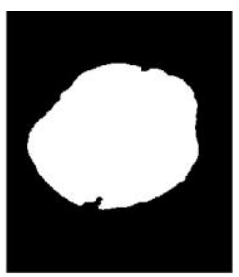

(b)

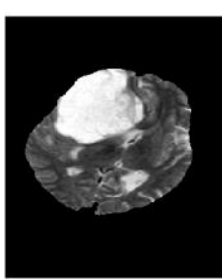

(c)

Figure 4. Process of extraction of skull tissues

Figure 4(a) shows the MR input image. Figure 4(b) depicts the mask which has been created to remove the extra cranial tissues. Figure 4(c) shows the skull extracted image which is obtained by the superimposition of Figure 4(a) and Figure 4 (b).

This procedure is repeated for all the images to make them extra cranial tissue- free images. Some of the skull extracted images are shown below in Figure 5 .

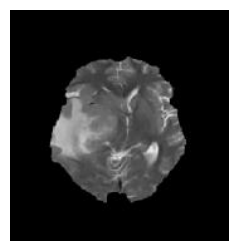

(a)meningioma

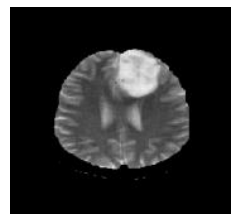

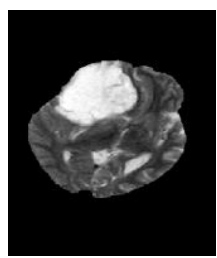

(b) astrocytoma

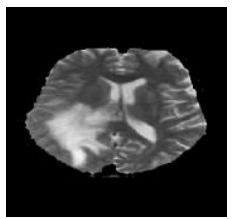

(c) glioma

(d) metastase

Figure 5. Sample skull extracted images from each tumor type.

Stage 2 results:

After removing the skull, the images are segmented using the fuzzy clustering algorithm. The same images are also segmented without removing the skull tissues. Their results are compared with the ground truth images. Figure 6 and Figure 7 shows the results of the fuzzy segmentation technique and the corresponding ground truth image.

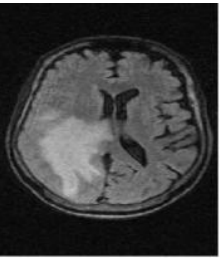

(a)

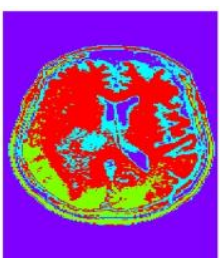

(b)

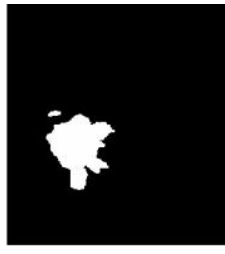

(c)
Figure 6. Segmented output of a MR image with extra cranial tissues

Figure 6(a) shows the original input image and Figure 6(b) shows the fuzzy segmented output with pseudo-colouring. The pixels represented by blue colour represent the tumor region. Figure 6(c) shows the corresponding ground truth image.

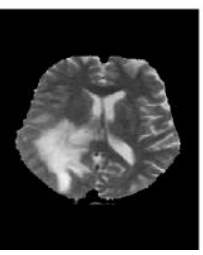

(a)

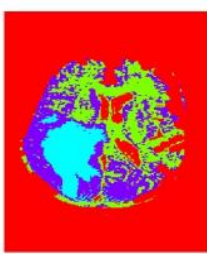

(b)

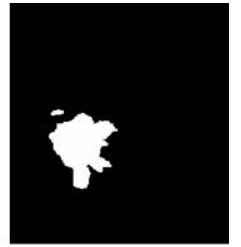

(c)
Figure 7. Segmented output of a MR image without extracranial tissues

Figure 7(a) shows the skull stripped input image and Figure 7(b) shows the fuzzy segmented output with pseudo-colouring. The pixels represented by blue colour represent the tumor region. Figure $7(\mathrm{c})$ shows the corresponding ground truth image.

A qualitative analysis of Figure 6(b) and Figure 7(b) clearly reveals the necessity for the removal of the extracranial tissues. Figure 7(b) closely resembles the ground truth which possesses high segmentation efficiency. The same process is repeated for all the images in the dataset. Table 2 shows the average segmentation efficiency for the images with extracranial tissues and images without extracranial tissues.

Table 2: Average segmentation efficiency for images with and without extracranial tissues

\begin{tabular}{|l|ll|}
\hline Image type & $\begin{array}{l}\text { Average } \\
\text { efficiency }\end{array}$ & Segmentation \\
\hline
\end{tabular}




\begin{tabular}{|lr|r|}
$\begin{array}{l}\text { MR images } \\
\text { extracranial tissues }\end{array}$ & with & $69.33 \%$ \\
\hline $\begin{array}{l}\text { MR images } \\
\text { extracranial tissues }\end{array}$ & without & $92.84 \%$ \\
\hline
\end{tabular}

Table 2 reveals the inferior nature of the MR images with extra cranial tissues. The segmentation efficiency is very low when compared with the images without extracranial tissues. A further analysis is made between the four different tumor types based on average segmentation efficiency. Table 3 shows the segmentation efficiency of four tumor types.

Table 3: Average segmentation efficiency for the different tumor type images without extracranial tissues

\begin{tabular}{|l|c|}
\hline Tumor type & $\begin{array}{l}\text { Average } \\
\text { efficiency }\end{array}$ \\
\hline Metastase & $95 \%$ \\
\hline Astrocytoma & $94.87 \%$ \\
\hline Glioma & $90.04 \%$ \\
\hline Meningioma & $91.44 \%$ \\
\hline
\end{tabular}

Experimental results reveal the superior results for all the tumor types. This analysis shows that the proposed methodology is applicable for all tumor types. This shows the significance of skull tissue removal irrespective of the input images. A bar chart representation of the performance analysis is shown in Figure 8 and Figure 9.

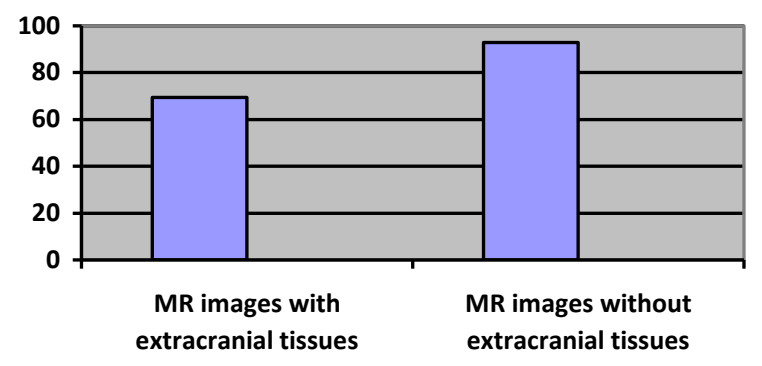

Figure 8. Bar diagram of the results of images with and without extracranial tissues

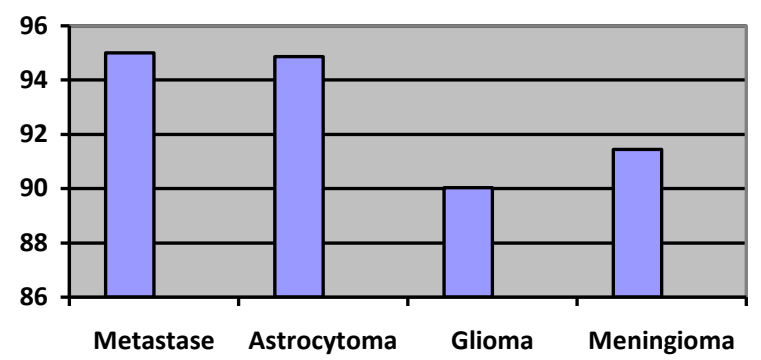

Figure 8. Bar diagram of the results of different tumor types

Thus, this experimental analysis clearly shows the significance of extracranial tissue removal techniques for abnormal MR brain image analysis.

\section{CONCLUSION}

The quantitative and the qualitative analysis of this work reveal the necessity for the removal of the extracranial tissues irrespective of the segmentation techniques used. Removal of the extracranial tissues guarantees high segmentation efficiency which is evident from the experimental results. This approach is highly efficient since the time taken for skull removal is also very less. As a future work, the proposed approach can be made an intensity independent technique to yield better segmentation efficiency.

\section{ACKNOWLEDGMENT}

The authors wish to thank the Management of M/s. Devaki. Scan Center, Madurai, Tamilnadu, India for their help regarding MRI data and validation.

\section{REFERENCES}

[1] Haack. E et.al. 1999. Magnetic Resonance Imaging, Physical Principles and Sequence Design. Weily-Liss, New York.

[2] Fletcher.H.L, Hall.L, Goldgof.D and Murtagh.F.R. 2001. Automatic segmentation of non-enhancing brain tumors in magnetic resonance images. Artificial Intelligence in Medicine. 21, 43-63.

[3] Hassan.K,et.al. 2009. 3D brain tumor segmentation in MRI using fuzzy classification, symmetry analysis and spatially constrained deformable models. Fuzzy Sytems and Archive. 160, 10, 1457-1473.

[4]Shan.Z.,Yue.G and .Liu.J. 2002. Automated Histogram-Based Brain Segmentation In T1-Weighted Three-Dimensional Magnetic Resonance Head Images. Neuroimage. 17, 1587-1598.

[5]Cardenas.V,.Ezekiel.A and Fenin,G.C. 2001. Reliability Of Tissue Volumes And Their Spatial Distribution For Segmented Magnetic Resonance Images. Psychiatry Research. 106, 193-205. [6]Stokking.R., Niceken,K.L and Viergever,M.A. 2001. Automatic Morphology Based Segmentation From MRI Data. Neuroimage. 12 ,726-738.

[7]Laperrire.N and Berstein.M. 1994. Radiotherapy For Brain Tumors. A Cancer Journal for Clinicians, 4, 96-108.

[8] Selvathi.D, et.al. 2005. MRI Image Segmentation Using Unsupervised Clustering Techniques. Proceedings of the Sixth International Conference on Computational Intelligence and Multimedia Applications. 105-110.

[9] Sanchez.D, et.al. 2005. Tree-dimensional technique for automatic brain segmentation of the ventricles based on optimal histogram thresholds of MRI. Proceedings of the 9th WSEAS International Conference on Computers. WSEAS Press, Stevens Point, Wisconsin, USA.

[10]Papageorgieu et.al. 2008. Brain tumor characterization using the soft computing technique of fuzzy cognitive maps. Applied Soft Computing. 8, 1, 820-828.

[11] Demir.C,et.al. 2005. IEEE/ACM Transactions on Computational Biology and Bioinformatics. 2, 3, 262-270.

[12] Garcia.C and Moreno.J. 2004. Kernel based method for segmentation and modeling of magnetic resonance images. Lecture Notes in Computer Science. 3315:636-645.

[13] Shubangi D.C, et.al. 2009. Support Vector Machine Classifier for Brain Tumor detection. International Conference on 
Advances in Computing, Communication and Control. ACM, NewYork. 444-448.

[14]Gering.D. 2003. Recognizing Deviations from Normalcy for Brain Tumor Segmentation. PhD thesis, MIT.

[15]Capelle.A, Colot.O, and Fernandez.C.M. 2004. Evidential segmentation scheme of multi-echo MR images for the detection of brain tumors using neighborhood information. Information Fusion. 5, 3, 203-216.

[16] Ruan.S, et.al. 2002. Fuzzy Markovian segmentation in application of magnetic resonance images. Computer Vision and Image Understanding. 85,1, 54-69.

[17]Taxt.T and Lundervold.A. 1994. Multispectral analysis of the brain using magnetic resonance imaging. IEEE Transactions on Medical Imaging. 13, 470-481.

[18] Siyal.M.Y and Yu.L. 2005. An intelligent modified fuzzy $c$ means based algorithm for bias estimation and segmentation of brain MRI. Pattern Recognition Letters. 26, 13, 2052-2062
[19] Gonzalez.R and Woods.R. 2002. Digital Image Processing. Pearson Education, $2^{\text {nd }}$ Edition.

[20] Thierry.G and Isabelle.B.2005. Segmentation of brain structures from MR images using morphological approaches. Medical Image Analysis. 162, 1-31.

[21] Jain.A.K. 1989. Fundamentals of Digital Image Procesing. Prentice-Hall, Inc. Upper Saddle River, NJ.

[22]Yang.Y and Zheng.Ch. 2005. Fuzzy C-means clustering algorithm with a novel penalty term for image segmentation. Opto-electronics review.13, 4, 309-315.

[23]MATLAB, User's Guide. 2002. The MathWorks.Inc. 